\title{
Retinal detachment with a giant bleeding cyst, simulating a uveal melanoma - a case report
}

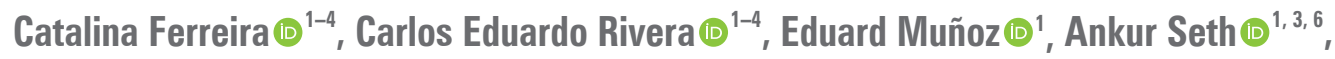 \\ Juan Carlos Aristizaba (1) ${ }^{2}$ \\ ${ }^{1}$ Collective Innovations, Cali, Colombia \\ ${ }^{2}$ Pontificia Universidad Javeriana, Cali, Colombia \\ ${ }^{3}$ GSR Medical Center, Cali, Colombia \\ ${ }^{4}$ GSR International, United States \\ ${ }^{5} U T$ Health San Antonio, San Antonio, United States \\ ${ }^{6}$ University of Tennessee Health Science Center, San Antonio, United States
}

\begin{abstract}
BACKGROUND: Pseudo-melanomas are a group of pathologies that can mimic melanoma. A retinal hemorrhagic macrocyst is a rare condition that can mimic choroidal melanoma. Diagnosing it accurately is important because of the difference in prognosis and treatment. Ocular ultrasound can be used to help differentiate between these similar conditions.
\end{abstract}

CASE PRESENTATION: We present a patient with a choroidal pseudo-melanoma diagnosed accurately and on time with the aid of an ocular ultrasound.

KEY WORDS: uveal melanoma; pseudomelanoma; ocular ultrasound

Ophthalmol J 2021; Vol. 6, 199-205

\section{INTRODUCTION}

Choroidal melanoma is the most frequently diagnosed intraocular malignant tumor in Caucasian adults. It rarely occurs in Latinos, although there are no published epidemiological studies in Latin America.

In the collaborative study of Ocular Melanoma [1] with eight years of follow-up of $6078 \mathrm{pa}$ tients recruited with sizeable choroidal melanoma (with more than $17.2 \mathrm{~mm}$ in baseline diameter and $9.5 \mathrm{~mm}$ in height), 1003 patients were found to be eligible, and $97 \%$ of those were non-Hispanic whites, with an average age of 60 years, with no preference for sex $[1,2]$.

Uveal melanoma represents less than $5 \%$ of all malignant melanomas [1] and leads to metastasis and death in $31 \%$ of the cases at five years [3].

Shields et al. [2] reported a study of 12,000 cases referred to the oncology service of the Wills Eye Hospital between 1978 and 2003, with suspected melanoma $14 \%$ of these cases were classified as and found to be pseudo-melanomas.

Hemorrhagic macrocyst of the retina is a rare disease that is occasionally associated with bleeding 
inside the cyst and is frequently misdiagnosed as choroidal melanoma.

Here we describe a case report of a patient with choroidal pseudomelanoma.

\section{CASE PRESENTATION}

A 74-year-old male patient presented with a one-year history of decreased vision in the right eye. He had a report of an ultrasound from a year ago with a diagnosis of retinal detachment. On the examination of the right eye, the vision was hand motion and presented with a month of the evolution of an appearance of a large, apparently subretinal, retropupillary mass (Fig. 1). An ultrasound had been performed at another institution where they reported an intraocular mass compatible with choroidal melanoma (Fig. 2).

The clinical diagnosis was of an intraocular tumor, probably a choroidal melanoma. The patient did not present with pain, complete vision loss, or other associated symptoms.

The ultrasound found a vitreous hemorrhage associated with a subtotal retinal detachment (Fig. 3). Evidence of a giant cyst, a lower temporal hemorrhage, and a level of hyphema inside (Fig. 4 and 5). On the 7 o'clock meridian, there was a small choroidal thickening in the posterior pole, with calcification points, high reflectivity, and an irregular structure, which was diagnosed as neovascularization (Fig. 6).

In the periphery of the lesion, a double membrane image was observed secondary to a division of the layers of the retina (Fig. 7).
Ultrasonic biomicroscopy revealed a flat retinal detachment and the periphery of the cyst with vitreous hemorrhage inside. Membranes with traction were also found between the periphery of the cyst and the ciliary body (Fig. 8).

The ultrasound-aided diagnosis was made of an old retinal detachment, associated with a giant hemorrhagic cyst with a level of hyphema inside and probable neovascularization.

In a 12-month ultrasound follow-up, the visual acuity was unchanged. A vitreous hemorrhage and an old retinal detachment were observed without the presence of the cyst (Fig. 9).

\section{DISCUSSION}

In $1964,19 \%$ of the enucleations due to uveal melanoma that were sent to the Institute of Pathology of the Armed Forces corresponded to a misdiagnosis. In 1984 it decreased to $1.4 \%$, in 1990 it was $0.4 \%$ and in 1998 it was reported to be only $0.3 \%[4]$.

The improvement in accuracy is due to increased use of ophthalmoscopy, better recognition of lesions that simulate melanomas, and the development of the ocular ultrasound [4].

Ocular ultrasound has helped support the diagnosis of melanoma even with unclear media, providing reliable measurements of the base and height of the tumor, evaluating the extra scleral extension, guiding for the correct location of the brachytherapy plate, and monitoring the conservative treatment of these patients.

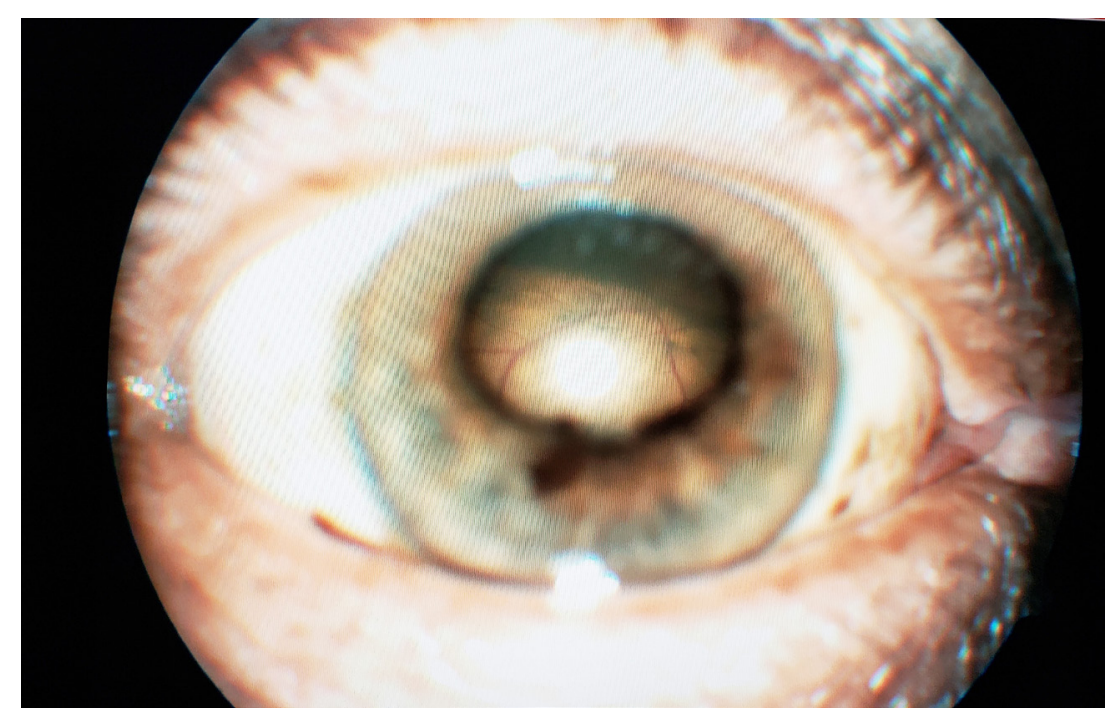

FIGURE 1. Bullous retinal detachment suggestive of a subretinal mass 


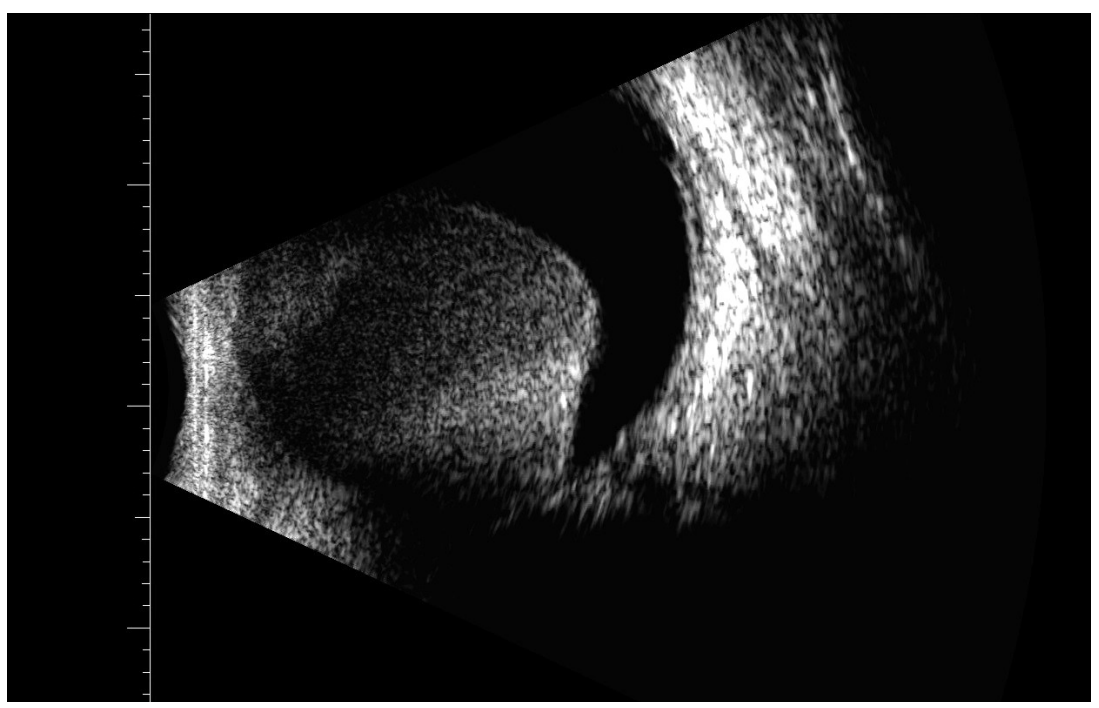

FIGURE 2. Nasal, longitudinal section with image suggestive of temporal mass

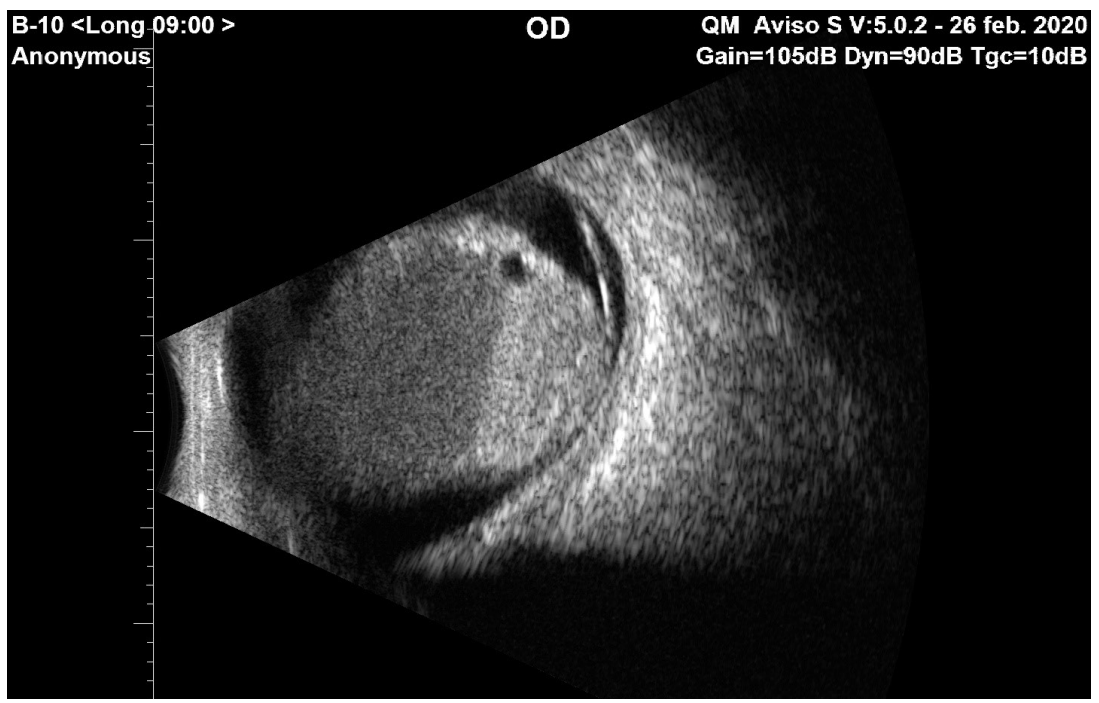

FIGURE 3. Flat retinal detachment image with a giant cyst. Note the cyst wall of irregular density, with a small cyst inside

In 2005, Shields et al. studied 1,739 patients classified as pseudo-melanoma and found 54 different pathologies. The most frequent diagnosis was choroidal nevus, which corresponded to $49 \%$ of cases, followed by a peripheral exudative hemorrhagic chorioretinopathy with $8 \%$ of cases, followed by congenital hypertrophy of the RPE at 6\% [2]. Some choroidal neoplasm pathologies can be misinterpreted, such as hemangiomas, leiomyomas, and choroidal metastases. There are non-neoplastic pathologies that can simulate choroidal melanoma. In less than $1 \%$ of cases, there have been ultrasonographic images of patients with hyper mature cataracts simulating melanoma. This occurs because the lens, seen in the extreme periphery, is shown as a dome shape mass image suggestive of melanoma and can be differentiated by performing an immersion ultrasound $[2,5]$.

Hemorrhagic retinal or choroidal detachments may occasionally manifest as a subchoroidal or pigmented subretinal mass and represent up to $5 \%$ of patients classified as pseudo-melanomas $[2,6]$. In these cases, it is essential to understand the cause of the bleeding. Highly reflective vascularized dome lesions can also be found in pathologies such as age-related macular degeneration, macroaneurysms, polypoidal choroidopathy, and trauma, among others $[2,6]$. 


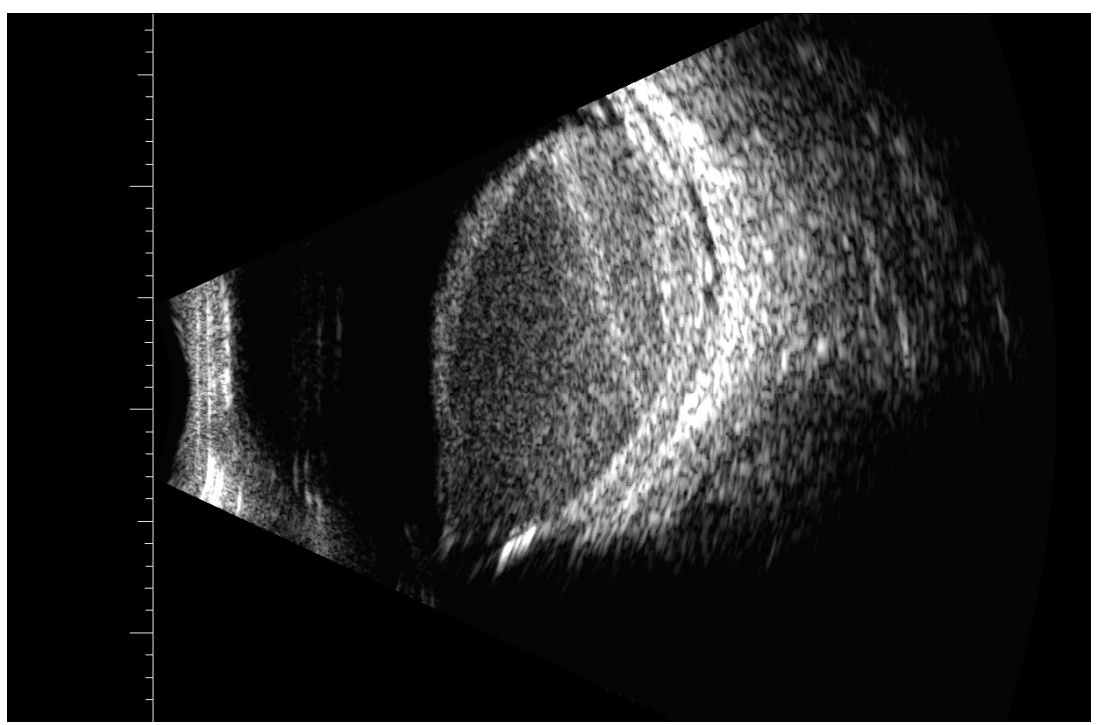

FIGURE 4. Cross-sectional image in the temporal and inferior quadrant. Imagen of a dome lesion is observed, with high-density content and a liquid level inside, compatible with hemorrhage and a level of hyphema

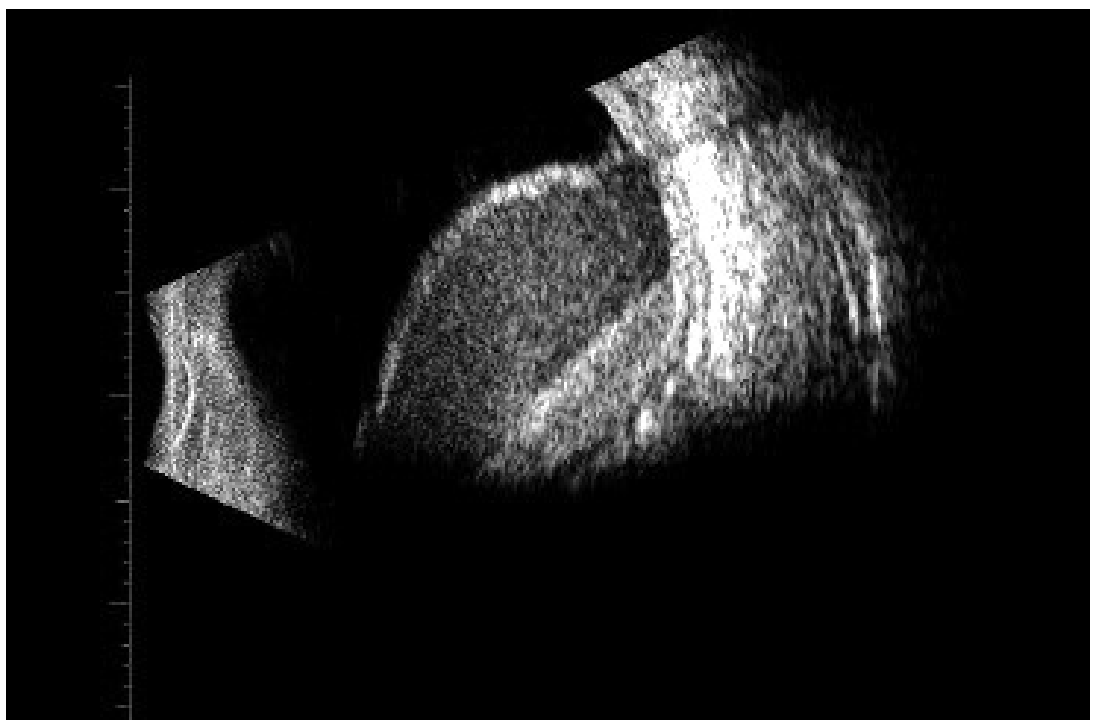

FIGURE 5. Hemorrhagic cyst. Compare figures 4 and 5 and note the movement of the hyphema with the eye movement

Although the clinical and ultrasound appearance of melanoma of comparable size to a subretinal or subchoroidal hemorrhage can be confusing, a subretinal hemorrhage tends to decrease in size on follow-up. However, it may persist for weeks to months.

Retinal macrocysts are a rare pathology that occurs in $3 \%$ of retinal detachments, usually more than three months old [7], and in some cases, they present with internal bleeding. There are few reports of cases confused with melanoma that have occasionally led to enucleation [7-9]. It is a pa- thology, usually asymptomatic, unilateral, with one or multiple cysts that are generally associated with chronic retinal detachment. A particular association has been seen with traumatic detachment and retinal dialysis [11], although our patient did not have a history of trauma.

The presence of blood in the cyst could be due to a rupture of the vessels that cover the cyst, the rupture of the vessels over the tear [11], or peripheral neovascularization $[7,10]$.

Echographically, melanoma looks like a solid mass with sound attenuation, an acoustic shadow, 


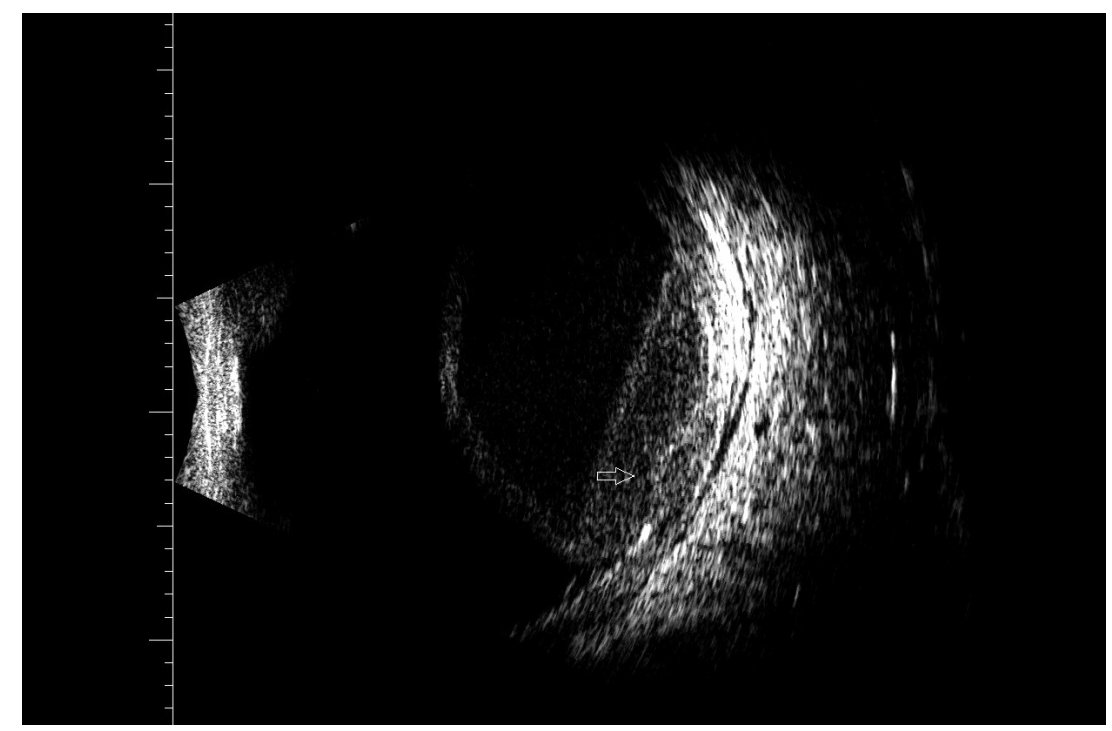

FIGURE 6. A cross-section of the posterior pole is observed. The cystic lesion and irregular choroidal thickening of high density in the ocular wall are compatible with a neovascular membrane. Note the calcium spots that create echoic shadowing

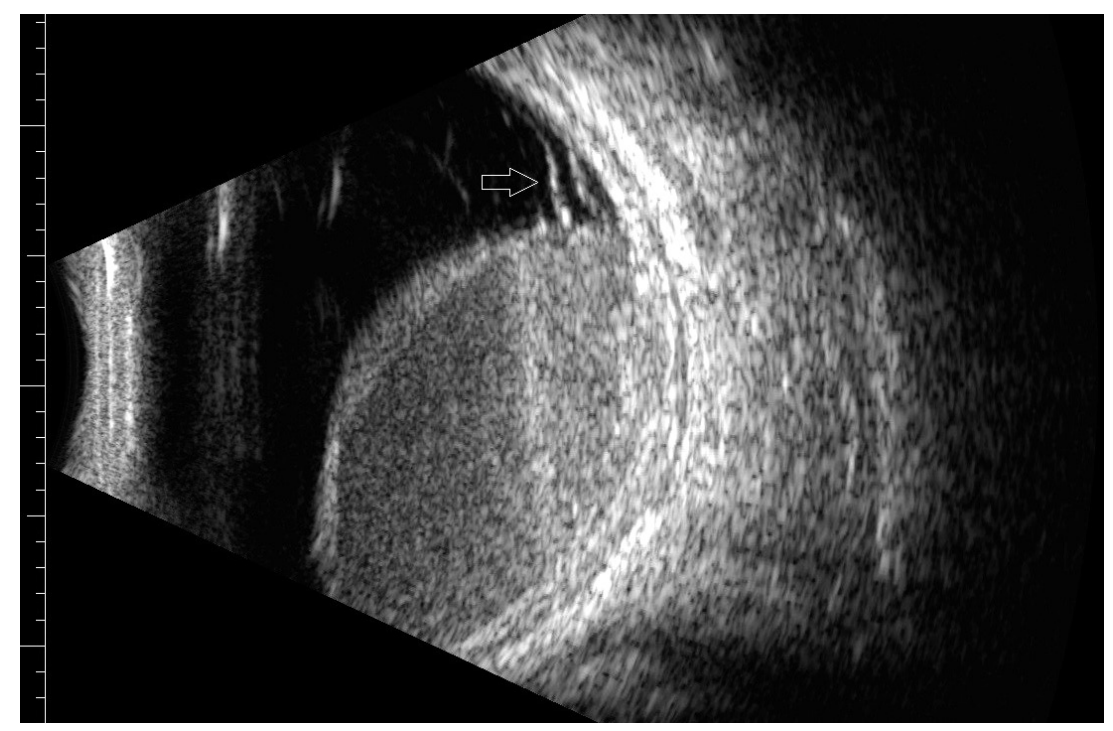

FIGURE 7. Retinal split in the periphery

and choroidal excavation. It can be in a dome or a mushroom shape $[1,11]$. Although the mushroom shape is highly suggestive of melanoma, it is known that it is not pathognomonic since there are several recent reports of mushroom-shaped choroidal metastases [1]. The internal vascularity is appreciable and can be associated with a hemorrhagic or non-hemorrhagic retinal detachment and vitreous hemorrhage. Mode A classically presents as a medium to low reflectivity (60-70\%) and a regular internal structure [1]. On rare occasions, the pres- ence of cysts inside or calcium on the surface of the tumor has been reported [13].

Ultrasonic biomicroscopy has been described as an accurate diagnostic means to determine the tumor's anterior margins and help with treatment planning [13].

Unlike melanoma, a retinal detachment on ultrasound imaging is seen as a very high-density membrane that inserts into the ora serrata and optic nerve. As signs of age, there may be folds secondary to retinal vitreous proliferation, calcium, and cysts. 


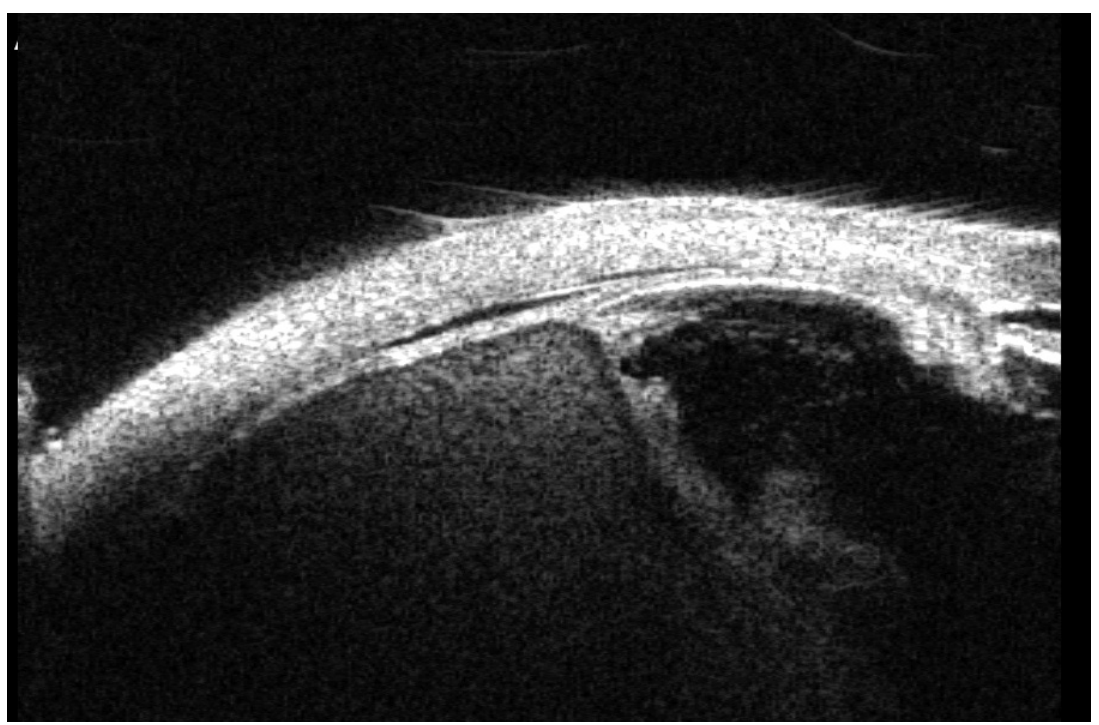

FIGURE 8. Ultrasound biomicroscopy with evidence of a flat retinal detachment. The peripheral end of the cyst is observed with evidence of bleeding inside. Membranes with traction are observed between the periphery of the cyst and the ciliary body

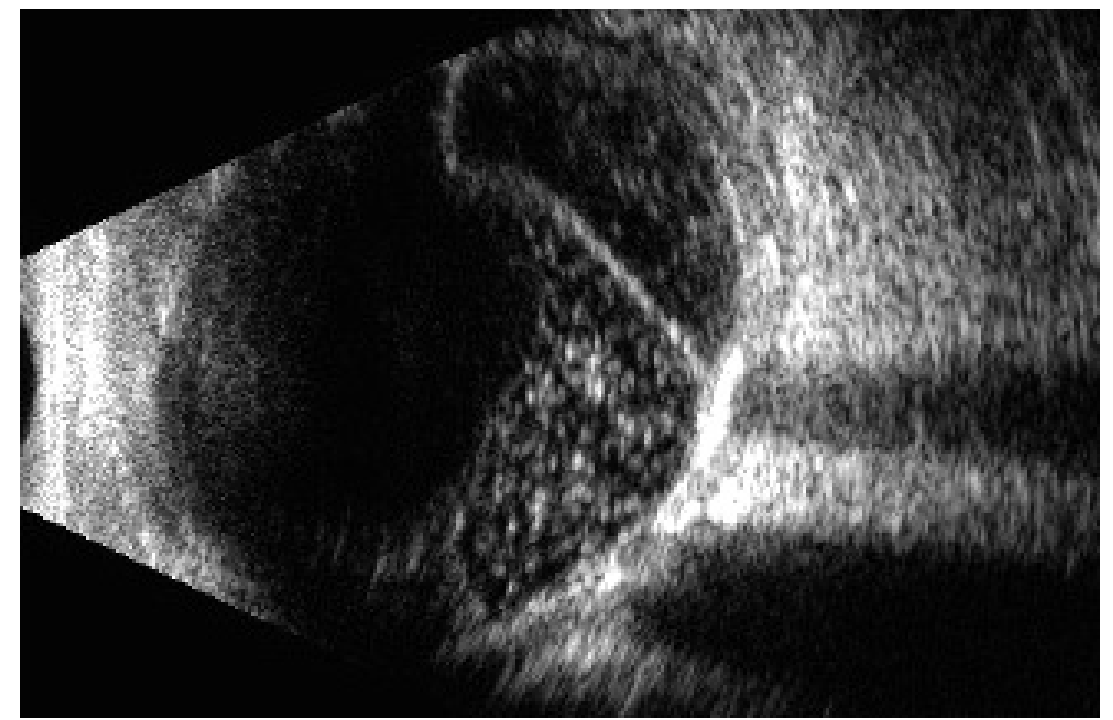

FIGURE 9. Control ultrasound at one year. Temporal inferior longitudinal section showing sub-vitreous hemorrhage, retinal detachment, and sub-retinal cholesterol. Ocular wall with calcium inside

The cysts, echographically, are observed as round lesions, dependent on the retina with very high reflectivity walls and in the cyst's periphery. In some patients, the division of the layers of the retina can be observed. [7] Occasionally, bleeding can be visualized in the interior of the cysts. This blood, like subretinal bleeding, does not coagulate but decants, producing a level of hyphema seen in our patient. It is noteworthy that the level of hyphema can be seen moving as the position of the patient's eye changes.

\section{CONCLUSIONS}

It is important to remember that the ideal management of uveal tumors must begin with a correct diagnosis. It is essential to properly differentiate between melanoma and pseudomelanoma because the treatment and prognosis of these pathologies are drastically different. Fortunately, thanks to the expertise of clinicians and the development of diagnostic technology, it is less likely to misdiagnose these patients [3]. 
Ocular ultrasound is a non-invasive and highly accurate method for differentiating choroidal melanoma from pseudomelanoma.

\section{REFERENCES}

1. The Collaborative Ocular Melanoma Study (COMS) randomized trial of pre-enucleation radiation of large choroidal melanoma I: characteristics of patients enrolled and not enrolled. COMS report no. 9. Am J Ophthalmol. 1998; 125(6): 767-778, doi: 10.1016/s0002-9394(98)00038-5, indexed in Pubmed: 9645715.

2. Shields JA, Mashayekhi $A$, Ra $S$, et al. Pseudomelanomas of the posterior uveal tract: the 2006 Taylor R. Smith Lecture. Retina. 2005; 25(6): 767-771, doi: 10.1097/00006982-200509000-00013, indexed in Pubmed: 16141866.

3. Kujala $E$, Mäkitie T, Kivelä T. Very long-term prognosis of patients with malignant uveal melanoma. Invest Ophthalmol Vis Sci. 2003; 44(11): 4651-4659, doi: 10.1167/iovs.03-0538, indexed in Pubmed: 14578381.

4. Boldt HC, Byrne SF, Gilson MM, et al. Collaborative Ocular Melanoma Study Group. The Collaborative Ocular Melanoma Study (COMS) randomized trial of pre-enucleation radiation of large choroidal melanoma I: characteristics of patients enrolled and not enrolled. COMS report no. 9. Am J Ophthalmol. 1998; 125(6): 767-778, doi: 10.1016/s00029394(98)00038-5, indexed in Pubmed: 9645715.

5. Shields CL, Pellegrini M, Kligman BE, et al. Ciliary body and choroidal pseudomelanoma from ultrasonographic imaging of hypermature cataract in 20 cases. Ophthalmology. 2013; 120(12): 2546-2551, doi: 10.1016/j.ophtha.2013.05.024, indexed in Pubmed: 23916484.
6. Shields CL, Salazar PF, Mashayekhi A, et al. Peripheral exudative hemorrhagic chorioretinopathy simulating choroidal melanoma in 173 eyes. Ophthalmology. 2009; 116(3): 529-535, doi: 10.1016/j. ophtha.2008.10.015, indexed in Pubmed: 19157563.

7. Mansour AM, Jaroudi MO. Hemorrhagic retinal macrocysts, simulating choroidal melanoma: a case report. Clin Ophthalmol. 2013; 7: 973-976, doi: 10.2147/OPTH.S45711, indexed in Pubmed: 23761961.

8. Zimmerman LE, Spencer WH. The pathologic anatomy of retinoschisis with a report of two cases diagnosed clinically as malignant melanoma. Arch Ophthalmol. 1960; 63: 10-19, doi: 10.1001/ archopht.1960.00950020012002, indexed in Pubmed: 13847542.

9. Ruiz RS. Hemorrhagic macrocyst of the retina. Mistaken for malignant melanoma of the choroid. Arch Ophthalmol. 1970; 83(5): 588-590, doi: 10.1001/archopht.1970.00990030588011, indexed in Pubmed: 5442149 .

10. Verdaguer $P$, Nadal J. Intraretinal cyst secondary to longstanding retinal detachment. Eur J Ophthalmol. 2012; 22(3): 506-508, doi: 10.5301/ ejo.5000034, indexed in Pubmed: 21928257.

11. Shields CL, Pellegrini M, Kligman BE, et al. Ciliary body and choroidal pseudomelanoma from ultrasonographic imaging of hypermature cataract in 20 cases. Ophthalmology. 2013; 120(12): 2546-2551, doi: 10.1016/i.ophtha.2013.05.024, indexed in Pubmed: 23916484.

12. Read R, Green R, Rao N. Metastatic adenocarcinoma with rupture through the bruch membrane simulating a choroidal melanoma. Am J Ophthalmol. 2001; 132(6): 943-945, doi: 10.1016/s00029394(01)01147-3, indexed in Pubmed: 11730672.

13. Maberly D, Pavlin C, McGowan H, et al. Ultrasound Biomicroscopic Imaging of the Anterior Aspect of Peripheral Choroidal Melanomas. American Journal of Ophthalmology. 1997; 123(4): 506-514, doi: 10.1016/s0002-9394(14)70176-x, indexed in Pubmed: 9124247. 